\title{
Explorative study of apple juice fluorescence in relation to antioxidant properties
}

\author{
Katarzyna Włodarska ${ }^{a}$, Katarzyna Pawlak-Lemańska ${ }^{a}$, Igor Khmelinskii ${ }^{\mathrm{b}}$, Ewa Sikorska ${ }^{\mathrm{a}, *}$ \\ ${ }^{a}$ Faculty of Commodity Science, Poznań University of Economics and Business, al. Niepodległości 10, 60-967 Poznań, Poland \\ ${ }^{\mathrm{b}}$ Universidade do Algarve, FCT, DQB and CIQA, Campus de Gambelas, Faro 8005-139, Portugal
}

\section{A R T I C L E I N F O}

\section{Article history:}

Received 15 April 2015

Received in revised form 11 March 2016

Accepted 1 May 2016

Available online 2 May 2016

\section{Keywords:}

Apple juice

Antioxidants

Fluorescence

Parallel factor analysis

Multivariate regression

\begin{abstract}
A B S T R A C T
Total fluorescence spectra (excitation-emission matrices, EEM) were recorded for a series of commercial apple juices, including clear and cloudy juices produced from concentrate, cloudy juices that were not from concentrate, and freshly squeezed juices. An exploratory study of the spectra with parallel factor analysis (PARAFAC) revealed three groups of fluorophores with different emission properties, and these properties were characterized by excitation/emission maxima at $270 / 315 \mathrm{~nm},(310,370) / 455 \mathrm{~nm}$, and $430 /(550,680) \mathrm{nm}$, respectively.

A regression analysis of the total fluorescence spectra arranged into three-way arrays using $\mathrm{N}$-way partial least squares regression methods (NPLS1 and NPLS2) and an analysis of the unfolded spectra by partial least squares methods (PLS1 and PLS2) revealed quantitative relations between the fluorescence and antioxidant properties of juices. The best models for the total phenolic contents and total antioxidant capacities were obtained by applying the NPLS1 method to the EEM. The model parameters were as follows: $R_{C V}^{2}=0.802, R P D=2.3$ for the total phenolic content and $R_{C V}^{2}=0.808$ and $R P D=2.3$ for the total antioxidant capacity. These results show the potential use of fluorescence spectroscopy for screening apple juices for their antioxidant properties.
\end{abstract}

๑ 2016 Elsevier Ltd. All rights reserved.

\section{Introduction}

Because of their widespread consumption, apples and apple juices are among the major dietary sources of phytochemicals, including phenolics. Phenolic compounds account for the metabolic activity and antioxidant properties of plant-based foods and for putative health benefits in humans. The consumption of apples and apple products has been positively associated with a reduction in the risk of developing several diseases (Candrawinata, Golding, Roach, \& Stathopoulos, 2013). Moreover, phenols affect the organoleptic properties of foods, including their colours, flavours, astringency, and hardness (Robbins, 2003).

Phenolic compounds are distributed differently throughout the apple fruit. The contents and profiles of phenolic compounds vary depending on the apple variety and environmental and postharvest factors (the fruit season, fruit maturity, light exposure, and storage) (Kalinowska, Bielawska, Lewandowska-Siwkiewicz,

\footnotetext{
* Corresponding author at: Faculty of Commodity Science, Poznan University of Economics and Business, al. Niepodległości 10, 61-875 Poznan, Poland.

E-mail addresses: katarzyna.wlodarska@ue.poznan.pl (K. Włodarska), kasia. lemanska@ue.poznan.pl (K. Pawlak-Lemańska), ikhmelin@gmail.com (I. Khmelinskii), ewa.sikorska@ue.poznan.pl (E. Sikorska).
}

Priebe, \& Lewandowski, 2014). Fresh apples and juices differ in their phenolic contents and composition. The processing of apples during juice production significantly reduces their phenolic contents and antioxidant activities. The phenolic content of an apple juice may also change during storage as a result of both oxidative and non-oxidative degradation (Candrawinata et al., 2013).

Apples contain various phenolic compounds, including hydroxybenzoic acids ( $\mathrm{p}$-hydroxybenzoic, protocatechuic, gallic, syringic, and gentisic acids), hydroxycinnamic acids and their derivatives (p-coumaric, caffeic, ferulic, and chlorogenic acids), flavonols (quercetin, which is present in glycosylated forms), dihydrochalcones (phloridzin and its derivatives), anthocyanids (cyanidines and their glycosides), monomeric flavanols (epicatechin and catechin), and oligomeric flavanols (procyanidins). Normally, phenolic antioxidants determine the antioxidant activity of apples, and the contribution of vitamin C is small (Kalinowska et al., 2014).

A variety of methods have been used to determine the phenolic contents and antioxidant activities of foods (Craft, Kerrihard, Amarowicz, \& Pegg, 2012; Ignat, Volf, \& Popa, 2011). HPLC coupled with detection by absorbance, mass spectrometry or fluorimetry is one of the most frequently used methods to quantify individual phenolics (Corradini et al., 2011; Pyrzynska \& Sentkowska, 2014). Other useful instrumental techniques involving separation include 
high-speed counter-current chromatography, supercritical fluid chromatography, gas chromatography, and capillary electrophoresis (Ignat et al., 2011).

In foods, the total phenolic content and antioxidant capacity are often quantified rather than the concentration of individual phenolic compounds. Despite their inherent limitations, these assays are often used in research (Craft et al., 2012; Ignat et al., 2011).

Several spectrophotometric methods are used to quantify the total phenolic contents and individual classes of phenolic compounds. Among these approaches, the Folin-Ciocalteu assay is used widely to determine the total phenolics (Ignat et al., 2011).

Several methods have been proposed for measuring the antioxidant activity of individual compounds and the antioxidant capacity of foods (Craft et al., 2012). These commonly used assays measure the Trolox equivalent antioxidant capacity (TEAC), oxygen radical absorbance capacity (ORAC), total radical-trapping antioxidant parameter (TRAP), ferric-reducing ability of plasma (FRAP) and 2,2-diphenyl-1-picrylhydrazyl radical cation (DPPH) assay (Craft et al., 2012). All these methods rely on measurements of the ability of a food or food component to scavenge specific free radicals or reduce other chemicals. The measured activity is calibrated against that of a reference compound, usually Trolox (a water-soluble derivative of vitamin E), gallic acid, or catechin.

Spectroscopic techniques coupled to chemometrics might provide an alternative to conventional methods in routine, highthroughput determinations of phenolic compounds and antioxidant capacities of foods. NIR and MIR spectroscopy coupled with chemometrics have been used to assess both the concentrations of the bioactive components and the total antioxidant capacities of foods (Lu \& Rasco, 2011). For example, NIR spectroscopy and multivariate regression have been used to predict the polyphenol contents of apples (Giovanelli, Sinelli, Beghi, Guidetti, \& Casiraghi, 2014; Pissard et al., 2013; Schmutzler \& Huck, 2014).

Some of the phenolic compounds are fluorescent, and their contributions to the autofluorescence of food products has been reported, including that of apple juices (Poryvkina, Tsvetkova, \& Sobolev, 2014; Seiden, Bro, Poll, \& Munck, 1996), wine (AiradoRodríguez, Durán-Merás, Galeano-Díaz, \& Wold, 2011; AiradoRodríguez, Galeano-Díaz, Durán-Merás, \& Wold, 2009), honey (Karoui, Dufour, Bosset, \& De Baerdemaeker, 2007; Lenhardt, Bro, Zeković, Dramićanin, \& Dramićanin, 2015; Sergiel, Pohl, Biesaga, \& Mironczyk, 2014) and strawberry fruits (Wulf et al., 2008). Recently, fluorescence spectroscopy coupled with multivariate regression was used to screen the antioxidant contents and the antioxidant capacities of coffee, peppermint extract and tomato paste (Orzel \& Daszykowski, 2014; Orzel, Stanimirova, CzarnikMatusewicz, \& Daszykowski, 2015). Synchronous fluorescence spectroscopy was used to determine the phenolic acids and scopoletin in brandies (Žiak, Sádecká, Májek, \& Hroboňová, 2014). These studies show that because of its high selectivity and sensitivity, fluorescence may be a valuable alternative to absorption spectroscopic techniques for quantifying the phenolic compounds in foods.

Several apple juice studies have successfully employed fluorescence. Fluorescence spectra were correlated with the soluble solid contents of apple juice (Seiden et al., 1996). Juices from two apple varieties were classified on the basis of their fluorescence (Seiden et al., 1996). This classification was also performed for apple juices that had been processed using different techniques (Poryvkina et al., 2014). Fluorescence was also correlated with newly formed products during the thermal processing of apple juices, supporting the usefulness of this parameter for monitoring the non-enzymatic browning of juice (Cohen, Birk, Mannheim, \& Saguy, 1998; Zhu, Ji, Eum, \& Zude, 2009).

To the best of our knowledge, there are no quantitative studies of apple juice fluorescence in relation to phenolic contents and antioxidant capacities. Thus, the aim of this study was to explore the relations between the natural fluorescence of apple juices and their total phenolic contents (TPC) and total antioxidant capacities (TAC). Spectrophotometric assays were used as reference methods to determine the TPC and TAC.

The total fluorescence spectra (excitation-emission matrices, or EEM) were recorded for each juice, and a parallel factor analysis (PARAFAC) was used in this exploratory study. Regression analyses using partial least squares (PLS) and $N$-way partial least squares (NPLS) were employed to investigate the relations between the fluorescence spectra of juices and TPC and TAC.

\section{Materials and methods}

\subsection{Reagents}

Folin-Ciocalteu reagent (Sigma-Aldrich, Steinheim, Germany) and $20 \%$ sodium carbonate (POCh, Gliwice, Poland) were used to determine the total phenolic contents, with gallic acid (SigmaAldrich, Steinheim, Germany) as a reference.

For the TEAC assay, the ABTS $^{+}$radical cation was generated using ABTS (2,2'-azino-bis[3-ethylbenzothiazoline-6-sulphonic acid] diammonium salt from Roche (Mannheim, Germany) and potassium persulphate from Fluka (Buchs, Switzerland). Trolox (6-hydroxy-2,5,7,8-tertamethylchroman-2-carboxylic acid) was purchased from Sigma-Aldrich (Steinheim, Germany) and used as a standard.

\subsection{Apple juices}

Thirty apple juices that are available on the Polish market were evaluated in this study. These samples included juices from concentrate that were clear and cloudy, and not-from-concentrate juices that were pasteurized naturally cloudy and freshly squeezed. Juices from 18 different producers were studied; some of these samples were studied in duplicate (the samples were taken from two different production batches). The studied samples include juices that were produced from concentrate, clear (group A - 11 samples: A1a, A1b, A2, A3a, A3b, A4a, A4b, A5a, A5b, A6a, and A6b) and cloudy (group B - 2 samples: B1 and B2), and notfrom-concentrate, including pasteurized naturally cloudy juices (group C - 13 samples: C1a, C1b, C2a, C2b, C3a, C3b, C4a, C4b, C5a, C5b, C6a, C6b, and C7) and freshly squeezed juices (group D - 4 samples: D1a, D1b, D2a, and D2b). Samples from the same producers are denoted with the same capital letter and number, and lower-case letters distinguish the different production batches.

\subsection{Determination of the chemical parameters}

\subsubsection{Total phenolic content (TPC)}

The TPC was determined according to Singleton and Rossi (1965), and the method was adapted to 48-well microplates. This method is based on the absorbance measurement of the reaction product at $765 \mathrm{~nm}$, which is used to quantify the reduction of Folin-Ciocalteu reagent. In brief, $0.010 \mathrm{~mL}$ of each juice sample was diluted $1: 2$ with water and mixed with $0.050 \mathrm{~mL}$ of the Folin-Ciocalteu reagent. After $3 \mathrm{~min}, 0.15 \mathrm{~mL}$ of $20 \%$ sodium carbonate and $0.79 \mathrm{~mL}$ of deionized water were added and the solution was mixed well. After $120 \mathrm{~min}$ in the dark at room temperature, the absorbance was measured using an EpochTH microplate spectrophotometer (BioTek). The TPC was expressed as mg of gallic acid equivalents per litre of juice ( $\mathrm{mg}$ GAE/L). These analyses were made at least in triplicate. 


\subsubsection{The total antioxidant capacity (TAC)}

The TAC of juices was determined using the TEAC assay according to Re et al. (1999), as described in detail in Gliszczyńska-Świgło et al. (2006). This method is based on the absorption decay of the $\mathrm{ABTS}^{+}{ }^{+}$radical cation at $734 \mathrm{~nm}$, with the increase of the juice/Trolox concentration. The TEAC value was calculated from the linear regression coefficient of the calibration curve for six dilutions of the juice, as calibrated against the linear regression coefficient of the Trolox curve. The ABTS ${ }^{+}$cation radical was generated by tracking the interaction of $7.7 \mathrm{mg}$ of ABTS that was dissolved in $1.8 \mathrm{~mL}$ of deionized water and $0.2 \mathrm{~mL}$ of $0.0069 \mathrm{~g} / \mathrm{mL}$ potassium persulphate. The cation was incubated in the dark at room temperature for $16 \mathrm{~h}$. The $\mathrm{ABTS}^{+}$cation radical working solution was diluted with methanol to an absorbance of 0.80 at $734 \mathrm{~nm}$. The absorbance was recorded 6 min after mixing $0.008 \mathrm{~mL}$ of juice with $0.792 \mathrm{~mL}$ of the ABTS ${ }^{+}$working solution. The TEAC value was expressed in mmol of Trolox per litre of juice ( $\mathrm{mM})$, with Trolox being used as a standard in this assay. These measurements were performed on a Spectronic Genesys 2 spectrophotometer (Milton Roy). All determinations were performed in triplicate.

\subsection{Fluorescence measurements}

The fluorescence spectra were recorded using a Fluorolog 3-11 spectrofluorometer (Spex-Jobin Yvon, France). The total fluorescence spectra (excitation-emission matrices, EEM) were obtained by recording the emission spectra in the $260-700 \mathrm{~nm}$ range with the excitation in the $250-500 \mathrm{~nm}$ range, at $10 \mathrm{~nm}$ steps in the excitation wavelength.

The excitation and emission slit widths were $3 \mathrm{~nm}$. The acquisition interval and the integration time were maintained at $1 \mathrm{~nm}$ and $0.1 \mathrm{~s}$, respectively. A reference photodiode detector at the excitation monochromator stage compensated for the source intensity fluctuations. The individual spectra were corrected for the wavelength-dependent response of the system.

Front-face geometry was used for undiluted samples in a $10 \mathrm{~mm}$ fused-silica cuvette. Samples of clear juices were measured directly, without any treatment; samples of cloudy juices were measured after centrifugation (15,000 rpm for $5 \mathrm{~min})$.

\subsection{Data analysis}

Pearson correlation coefficients were calculated to test for the correlations between the individual analytical parameters.

Parallel factor analysis (PARAFAC) was used to break the EEM into the contributions of the individual fluorescent components. PARAFAC is a generalization of a bilinear PCA that is expanded into three dimensions. Each PARAFAC component consists of one score vector and two loading vectors. The loading vectors correspond to the excitation and emission spectra of the individual components. The score vector contains information about the relative contribution of each component to each of the sample EEM included in the model (Andersen \& Bro, 2003).

A three-way data array with a total of $30 \times 26 \times 431$ components (number of samples $\times$ number of excitation wavelengths $\times$ number of emission wavelengths) was used in the PARAFAC analysis. The Rayleigh scattering contributions to the EEM were removed by inserting the missing values into the bands centred on the wavelength identity line and the $2 \lambda_{\text {ex }}=\lambda_{\text {em }}$ line. Non-negativity constraints were applied to the excitation and emission spectra and the concentrations. The core consistency diagnostic (CORCONDIA) was used for finding the optimal number of components in the PARAFAC models (Andersen \& Bro, 2003).

\subsubsection{Regression analysis}

Partial least squares regression (PLS) and $N$-way partial least squares regression (NPLS) were used to establish the calibration models between the fluorescence data (the $X$ matrix) and the chemical parameters (the $Y$ matrix).

The PLS method models both the $X$ - and $Y$-matrices simultaneously, finding the latent variables in $X$ that best predict the latent variables in $Y$ (Wold, Sjöström, \& Eriksson, 2001). The $N$-way PLS is an extension of the two-way PLS regression to the multi-way data, where the independent parameter set is an array of more than two dimensions.

PLS was used to model the relation between the total fluorescence spectra (EEM), which is held in an unfolded array, and the chemical data. The $X$ data included the entire EEM, which was arranged into a $30 \times 11,206$ matrix (number of samples $\times$ the number of emission wavelengths times the number of excitation wavelengths).

$N$-way PLS was used to model the relation between the total fluorescence (EEM) and the chemical data. The $X$ data consisting of the entire EEM were arranged into three-way arrays with a total of $30 \times 26 \times 431$ elements (number of samples $\times$ number of excitation wavelengths $\times$ number of emission wavelengths). The Rayleigh scattering contributions to the EEM were removed by inserting the missing values into the bands centred on the wavelength identity line and the $2 \lambda_{\text {ex }}=\lambda_{\text {em }}$ line.

The data pre-treatment included mean-centring over the first mode (over the sample set). Similar to the PLS1 regression, the $Y$ data for the $N$-way PLS1 analysis contained the values of an individual chemical parameter (TPC or TAC). Additionally, PLS2 and NPLS2 analyses were performed, modelling the two analytical parameters simultaneously.

Full leave-one-out cross-validation was applied to all of the regression models. The regression models were evaluated using the determination coefficient $\left(\mathrm{R}^{2}\right)$ and the root mean-square error of the cross-validation (RMSECV) as the term for indicating the prediction error of the model. The optimal number of components was chosen as the minimum for the plot of the RMSECV as a function of the number of components. The predictive ability of the models was evaluated by finding the ratio of the standard deviation for the reference data to the RMSECV, which was designated RPD. The RPD provides a means for standardizing the RMSECV and evaluating the robustness of the model (Nicolaï et al., 2007).

The data analysis was performed using Solo v. 5.0.1 software (Eigenvector Research Inc., USA).

\section{Results and discussion}

\subsection{Total phenolic contents and total antioxidant capacities of the apple juices}

The thirty apple juice samples studied here included clear (group A) and cloudy (group B) juices produced from concentrate, pasteurized naturally cloudy (group C) juices that were not produced from concentrate, and freshly squeezed juices (group D). The mean values, ranges and standard deviations of the total phenolic content (TPC) and the total antioxidant capacity (TAC) of the studied samples are presented in Table 1.

The antioxidant content and the antioxidant capacity that were determined using standard spectrophotometric methods showed significant variations within the sample set. The juices exhibit significant between-group variability in the studied parameters when the mean values were considered. However, the phenolic compound content and capacity varied significantly even within each of the groups. The observed variability likely resulted from both the production methods and the raw material composition. 
Table 1

The total phenolic content (TPC) and the total antioxidant capacity (TAC) of the apple juices under study ( $n=30$ samples).

\begin{tabular}{|c|c|c|c|c|c|c|c|}
\hline \multirow[t]{2}{*}{ Juices } & \multirow{2}{*}{$\begin{array}{l}\text { Number of } \\
\text { samples }\end{array}$} & \multicolumn{3}{|c|}{ TPC (mg GAE/L) } & \multicolumn{3}{|l|}{ TAC $(\mathrm{mM})$} \\
\hline & & Range & Mean & SD & Range & Mean & SD \\
\hline All & 30 & $174-1925$ & 552 & 403 & $1.1-16.7$ & 4.6 & 3.8 \\
\hline Clear from concentrate $(\mathrm{A})$ & 11 & $193-602$ & 385 & 135 & $1.1-5.0$ & 2.4 & 1.1 \\
\hline Cloudy from concentrate (B) & 2 & 386,491 & 439 & - & $4.4,5.5$ & 5.0 & - \\
\hline Cloudy not from concentrate (C) & 13 & $174-1925$ & 746 & 542 & $1.4-16.7$ & 6.7 & 4.8 \\
\hline Freshly squeezed (D) & 4 & $275-720$ & 436 & 195 & $2.2-8.2$ & 4.0 & 2.8 \\
\hline
\end{tabular}

The lowest mean values of the TPC and TAC were obtained for the clear juices from concentrate (group A) in comparison with the cloudy juices (groups B, C, and D).

The highest values for the studied parameters were recorded for cloudy juices that were not made from concentrate (group C), with some results even exceeding those reported in the literature (Kahle, Kraus, \& Richling, 2005). Several producers declared that vitamin $\mathrm{C}$ had been added to some juices. Thus, the added vitamin $C$ may have contributed to the high values observed for TAC and may have affected the TPC values. It should be noted that the TPC assay, which uses the Folin-Ciocalteu reagent, is subject to interference, particularly from readily reducible compounds. Ascorbic acid (vitamin $\mathrm{C}$ ) is therefore a confounding factor in the analysis of wine and most fruit (Craft et al., 2012).

Published data confirm the substantial variability in the quantity of phenolic antioxidants in apple juices as determined by HPLC. For example, the total phenolic contents found in laboratoryproduced juices were $1044.4 \mathrm{mg} / \mathrm{L}$ and $698.5 \mathrm{mg} / \mathrm{L}$ in Champion cloudy and clear juices, and $472.2 \mathrm{mg} / \mathrm{L}$ and $250.1 \mathrm{mg} / \mathrm{L}$ in Idared cloudy and clear juices, respectively (Oszmiański, Wolniak, Wojdyło, \& Wawer, 2007). For comparison, the total polyphenol contents in commercial clear juices ranged from 63.84 to $163.35 \mathrm{mg} / \mathrm{L}$ (Gliszczyńska-Świgło \& Tyrakowska, 2003). In another study of commercial apple juices, the total polyphenol contents of cloudy juices (21 samples) varied between 152 and $459 \mathrm{mg} / \mathrm{L}$, whereas in clear apple juices (3 samples), the contents ranged from 110 to $173 \mathrm{mg} / \mathrm{L}$ (Kahle et al., 2005).

A positive correlation was obtained between the TPC and TAC, with an estimated Pearson correlation coefficient of 0.944 . The observed relation between TPC and TAC for apple juices is in accordance with the literature data, indicating that the antioxidant properties of the apple juices are primarily determined by their phenolic contents (Gardner, White, McPhail, \& Duthie, 2000; Gliszc zyńska-Świgło \& Tyrakowska, 2003).

\subsection{Fluorescence of apple juices}

\subsubsection{Total fluorescence spectra}

Fig. 1 shows the EEM of four apple juices that represent each of the studied categories. Note that similar characteristic emission patterns are observed in all of the presented spectra. Specifically, four emission bands are present, and they have excitation/ emission maxima in the following ranges: $260-279 / 313-342 \mathrm{~nm}$, $308-320 / 438-451 \mathrm{~nm}, \quad 364-389 / 451-463 \mathrm{~nm}$, and 376-440/ $486-548 \mathrm{~nm}$. The individual juices differ in terms of the exact positions of the maxima and the intensities of the particular bands. Moreover, a fifth long-wavelength band is observed with the 415-418/678-681 nm excitation/emission maxima in some of the cloudy juices only.

\subsubsection{PARAFAC of the total fluorescence spectra}

A more detailed insight into the fluorescence characteristics of the entire sample set was provided by a PARAFAC analysis of the EEM data. The objective of this analysis was to resolve the fluorescence landscapes into the contributions of the individual fluores- cent components. Based on the core consistency and visual inspection of both the residuals and the loadings, an optimal PARAFAC model was identified as having three components (92.5\% of the variance was explained, with a 79.0 core consistency value). Fig. 2 shows the excitation and emission loadings of the three extracted components and their respective score plots.

The first component had excitation/emission maxima at $270 / 315 \mathrm{~nm}$. The second PARAFAC component was characterized by excitation spectrum with two bands displaying maxima at 310 and $370 \mathrm{~nm}$ and an emission band with a maximum at $455 \mathrm{~nm}$. The third PARAFAC component exhibited a broad excitation spectrum with its maximum at $430 \mathrm{~nm}$ and emission with its maximum at $550 \mathrm{~nm}$. An additional narrow band with a maximum at $680 \mathrm{~nm}$ was observed at the long-wavelength slope of the first band. Based on these results, it appears that the extracted PARAFAC components represented groups of chemical compounds with similar fluorescence properties rather than individual compounds.

The contributions of each of the three PARAFAC components to the spectra of the individual juices are shown in the score plots (Fig. 2c and d). The PARAFAC score values were plotted against one another (component 1 in comparison with 2, and component 2 in comparison with 3 ) to compare the fluorescence patterns of the studied juices. The clear juices from concentrate (group A) were characterized by the low-to-intermediate contribution of components 1, 2 and 3 relative to that of the other samples. The cloudy juices from concentrate (group B) and not-from-concentrate (group C) presented an intermediate contribution of component 1 and intermediate-to-high contributions of components 2 and 3. The fresh juices (group D) made an intermediate-to-high contribution of component 1 and relatively low contributions of components 2 and 3.

The PARAFAC score values allowed only limited discrimination among the juices according to their production method. Namely, the fresh juices (group D) were discriminated from the other three groups of juices (A, B, and $C$ ) in the plane that was defined by the first and second components. It should be noted that the juice properties are determined not only by processing but also by the raw materials; as we have stressed, similarly processed juices may present different antioxidant properties.

\subsubsection{Fluorescent components in the apple juices}

The assignment of the specific fluorescence bands is only tentative, and it is based on published data. Many of the native food components exhibit fluorescence (Christensen, Nørgaard, Bro, \& Engelsen, 2006). Apples contain several groups of compounds that are fluorescent, including aromatic amino acids, phenolics, and vitamins $B_{2}$ and $B_{6}$ (Feliciano et al., 2010; Wu et al., 2007). Chlorogenic acid is the leading phenolic compound found in apple juices. Other phenolics that have been reported in apple juices include caffeic acid, p-coumaric acid, ferulic acid, catechin, epicatechin, procyanidins (B1, B2, C1 trimer), rutin, phloridzin, quercetin, isoquercitrin, and hyperin (Gliszczyńska-Świgło \& Tyrakowska, 2003; Kahle et al., 2005; Karaman, Tütem, Sözgen Başkan, \& Apak, 2010). 

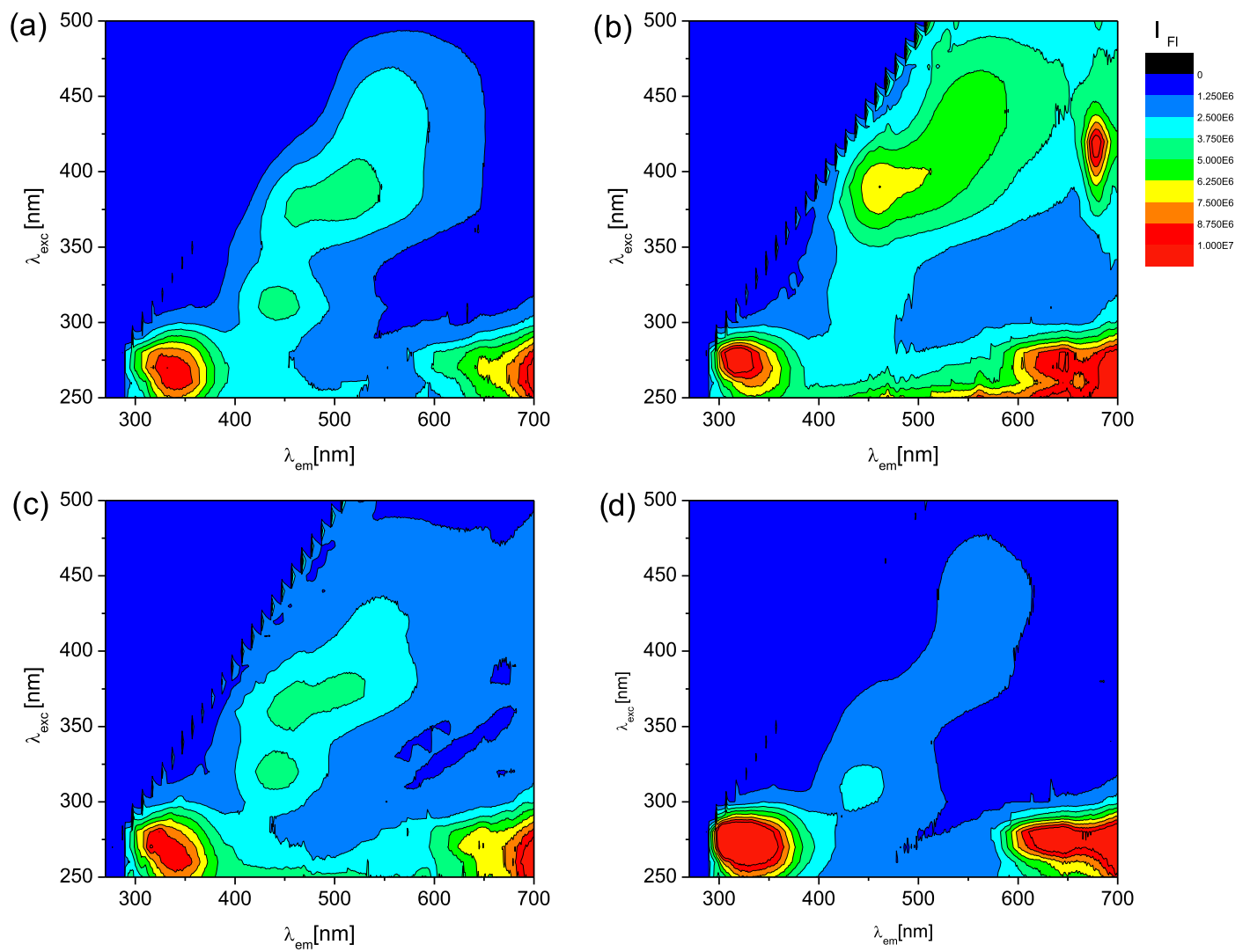

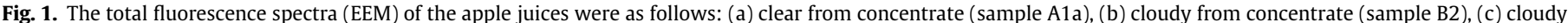
not from concentrate (sample C5a), and (d) freshly squeezed (sample D2a).
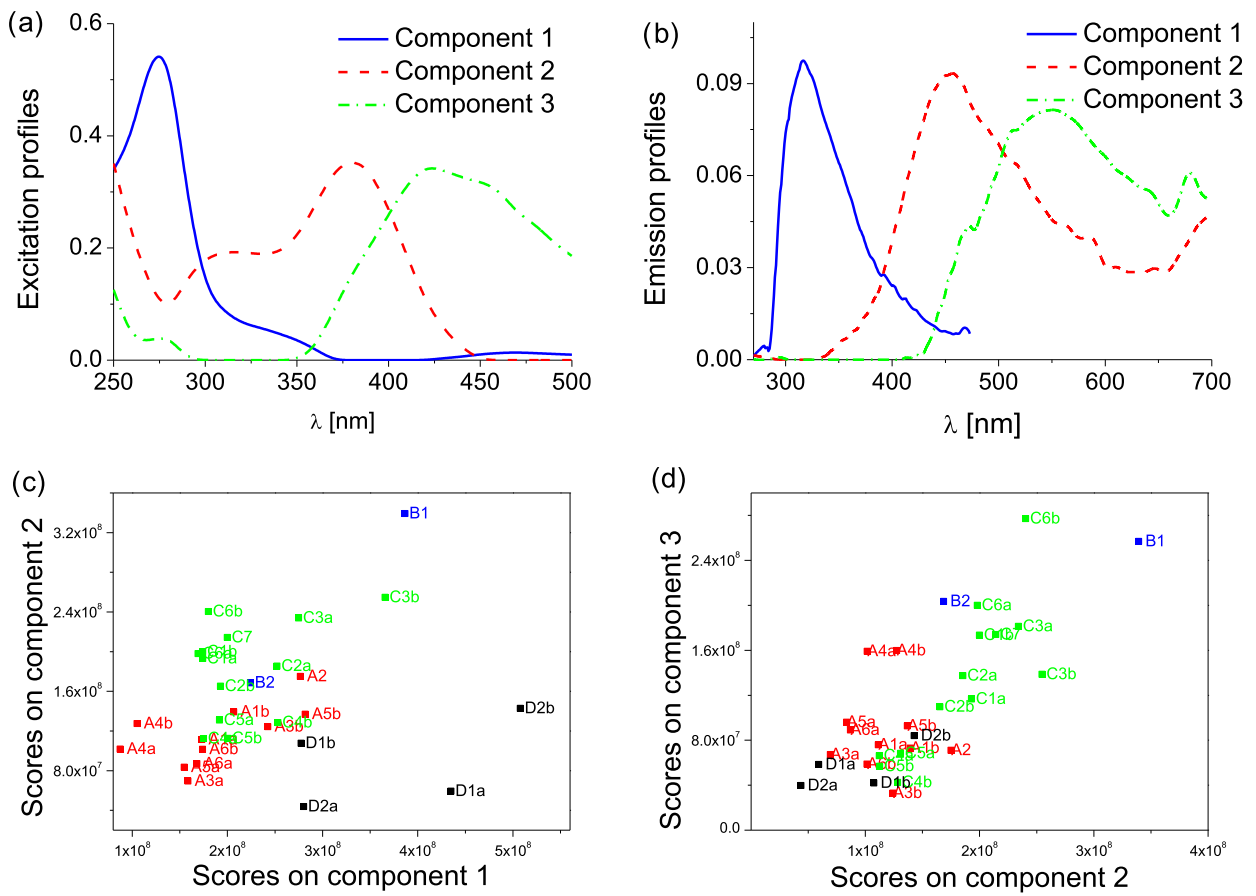

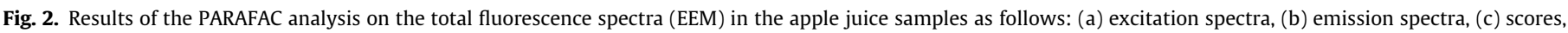
component 1 in comparison with component 2 and (d) scores, component 2 in comparison with component 3.

The first PARAFAC component had its excitation/emission maxima at $270 / 315 \mathrm{~nm}$. It should be noted that aqueous tyrosine solutions have excitation/emission maxima at 276/302 nm, and those of phenylalanine are 258/284 nm (Christensen et al., 2006). The presence of tyrosine and phenylalanine was reported in apples (Wu et al., 2007). Flavanols, including catechin and epicatechin, 
emit at 317 and $316 \mathrm{~nm}$ when excited at 279 and $280 \mathrm{~nm}$, respectively (Airado-Rodríguez et al., 2009).

The second PARAFAC component had an excitation spectrum with two bands centred at 310 and $370 \mathrm{~nm}$ and an emission band at $455 \mathrm{~nm}$. According to the published data (Airado-Rodríguez et al., 2009; Airado-Rodríguez et al., 2011), the emission maxima of many different phenolic compounds are all observed in approximately the same spectral range. The excitation/emission maxima of chlorogenic acid were observed at 245, 310/445 nm (Mazina, Vaher, Kuhtinskaja, Poryvkina, \& Kaljurand, 2015). When excited at $260 \mathrm{~nm}$, the emission maxima of the caffeic, ferulic and pcoumaric acids were reported at 426, 400 and $422 \mathrm{~nm}$, respectively (Airado-Rodríguez et al., 2011). The emission maximum for quercetin was reported at $400-420 \mathrm{~nm}$, with excitation at $260-262 \mathrm{~nm}$ (Airado-Rodríguez et al., 2011) or at $480 \mathrm{~nm}$ with excitation at $427 \mathrm{~nm}$ in tartrate buffer $(\mathrm{pH}=7)$ and $13 \%$ ethanol (Airado-Rodríguez et al., 2009). Another study found that quercetin fluorescence is pH-dependent, with dual emissions observed in aqueous solutions at $\mathrm{pH}=5$; weak fluorescence with maxima at $455 \mathrm{~nm}$ and $521 \mathrm{~nm}$ was attributed to the normal form and the tautomer formed in the excited-state proton transfer, respectively (Mezzetti, Protti, Lapouge, \& Cornard, 2011).

The excitation spectrum of the second PARAFAC component may also correspond to the absorption spectra of the phenolic compounds. Phenolic acids have absorption maxima in the 200$290 \mathrm{~nm}$ range, and because of an additional conjugation, hydroxycinnamic acids and their derivatives have an additional broad absorbance band, extending from 270 to $360 \mathrm{~nm}$ (Robbins, 2003). In addition, the typical UV-vis spectra of the flavonoids include two absorbance bands in the $350-385 \mathrm{~nm}$ and $250-290 \mathrm{~nm}$ ranges (Tsimogiannis, Samiotaki, Panayotou, \& Oreopoulou, 2007).

The third PARAFAC component exhibited a broad excitation spectrum with a maximum at $430 \mathrm{~nm}$ and an emission with a maximum at $550 \mathrm{~nm}$; when there was a second narrow band on the long-wavelength slope of this band, its maximum was $680 \mathrm{~nm}$ (Fig. 2a and b).

The fluorescence that occurred when the excitation/emission values were $400 / 493 \mathrm{~nm}$ (Cohen et al., 1998) and 400/476 nm (Zhu et al., 2009) was associated with the products of the nonenzymatic browning of the apple juices. The aqueous solution of riboflavin exhibits fluorescence at $448 / 518 \mathrm{~nm}$ in excitation/ emission (Christensen et al., 2006). Moreover, quercetin tautomer fluorescence may also contribute to this component, as indicated earlier (Mezzetti et al., 2011). The additional narrow emission band at the long-wavelength slope of the third PARAFAC component corresponds to chlorophyll, with its maximum at $680 \mathrm{~nm}$.

The emissions spectra of the pure compounds recorded in pure solvents may differ from those recorded in a food product because fluorescence is highly sensitive to the local environment and is affected by the matrix properties. This situation is complicated still further by the fact that the contributions of the individual components to the overall fluorescence are determined by both their concentrations and their fluorescence quantum yields (Christensen et al., 2006). Therefore, only a tentative attribution of the specific emission bands was possible, based on the data presented here.

\subsection{Multivariate calibration}

Multivariate regression was used to model the relations between the fluorescence and the antioxidant properties of the juices (TPC and TAC) in a quantitative fashion. All the samples $(n=30)$ were used in the analysis. The statistics for the calibration data set is shown in Table 1 . The regression was performed using NPLS for the analysis of the EEM that was arranged into a threeway array. PLS was used to analyse the unfolded EEM. Two different approaches were used, and they were based on PLS1 and NPLS1
Table 2

Characteristics of the regression models for the prediction of the total phenolic contents (TPC) and the total antioxidant activities (TAC) of the apple juices ( $n=30$ samples).

\begin{tabular}{|c|c|c|c|c|c|c|}
\hline \multirow[t]{2}{*}{ PLS model } & \multicolumn{3}{|c|}{ Calibration } & \multicolumn{3}{|c|}{ Cross-validation } \\
\hline & LV & $\mathrm{R}^{2}$ & RMSEC & $\mathrm{R}^{2}$ & RMSECV & RPD \\
\hline \multicolumn{7}{|l|}{ TPC } \\
\hline EEM NPLS 1 & 10 & 0.959 & 78.9 & 0.802 & 179 & 2.3 \\
\hline EEM NPLS 2 & 9 & 0.931 & 104 & 0.639 & 245 & 1.6 \\
\hline Unfolded EEM PLS1 & 8 & 0.950 & 88.3 & 0.737 & 203 & 2.0 \\
\hline Unfolded EEM PLS2 & 7 & 0.907 & 120 & 0.602 & 256 & 1.6 \\
\hline \multicolumn{7}{|l|}{ TAC } \\
\hline EEM NPLS 1 & 6 & 0.923 & 1.07 & 0.808 & 1.68 & 2.3 \\
\hline EEM NPLS 2 & 9 & 0.937 & 0.95 & 0.753 & 1.91 & 2.0 \\
\hline Unfolded EEM PLS1 & 8 & 0.956 & 0.76 & 0.749 & 1.90 & 2.0 \\
\hline Unfolded EEM PLS2 & 7 & 0.935 & 0.96 & 0.729 & 1.98 & 1.9 \\
\hline
\end{tabular}

LV - number of latent variables used for PLS, $\mathrm{R}^{2}$ - determination coefficient, RMSEC RMSECV - root mean square errors of calibration and cross validation in original units: mg GAE/L for TPC, mM for TAC, RPD - residual predictive deviation.

that modelled each of the analytical parameters individually, and on PLS2 and NPLS2 that analysed all of the parameters simultaneously. The pre-processing methods (smoothing, first derivative) provided no pronounced improvement in the regression results; therefore, the raw spectra without any preprocessing were used in all the analyses. Table 2 presents the results of the multivariate calibrations.

The models were evaluated on the basis of the RMSECV and the determination coefficient, $\mathrm{R}^{2}$. The usability of the models for predicting new samples was evaluated on the basis of the RPD value that provides a way to standardize the predictive accuracy (Nicolaï et al., 2007).

All of the calibration models confirm the correlation between the fluorescence and the parameters under study. The models that were obtained to predict the TPC and TAC usually had very similar prediction abilities. The performance of the PLS1 and NPLS1 models was generally better than that of their respective PLS2 and NPLS2 models. The performance of the NPLS1 and NPLS2 models was also better than that of the PLS1 and PLS2 models.

The optimal models for the TPC and TAC were based on the EEM, which was arranged into a three-way array and analysed using NPLS1. Both of these models had RPD $=2.3$, corresponding to an approximate quantitative prediction accuracy (Nicolaï et al., 2007). Thus, these models may at least be used in the juice screening for the appropriate TPC and TAC values.

The regression models for TPC and TAC included a relatively high number of latent variables, likely for several reasons. Firstly, the TPC that was estimated by the Folin-Ciocalteu assay quantifies a wide group of phenolic compounds, likely with diverse fluorescent properties. In accounting for the limited specificity of this method, non-phenolic and non-fluorescent compounds may also contribute to the result (e.g., the already mentioned vitamin C). Therefore, modelling TPC on the basis of fluorescence is quite complex and involves more latent variables. Similarly, complex calibration models may also be expected for the TAC values, which are estimated from the fluorescence spectra indirectly, by using their correlation with the TPC values.

\section{Conclusions}

In summary, the natural fluorescence of the apple juice was correlated with its antioxidant properties. The front-face EEM spectra provided overall emissions characteristics of the juice samples. PARAFAC analysis uniquely separated the three groups of fluorescent components, which contributed to the emissions of the individual juices differently. Some characteristic emission patterns 
surfaced in groups of similarly processed juices; however, the juice properties are strongly affected by those of the raw material, apart from the method used for their preparation. The PLS and NPLS regressions revealed the quantitative relation of the phenolic antioxidant content and antioxidant capacity with the fluorescence spectra.

The results and discussion presented here indicate directions for further studies, which should address several issues, including the unambiguous identification of the origin of the fluorescence bands, correlation of the fluorescence with the content of individual phenolic compounds, and need for additional tests on a larger number of samples to validate the prediction models.

Finally, we conclude that front-face fluorescence in association with chemometric analysis is a promising technique for the rapid screening of apple juices for antioxidant contents and antioxidant capacities.

\section{Acknowledgement}

We thank Prof. Anna Gliszczyńska-Świgło for the protocols for determining the given analytical parameters according to the reference methods.

\section{References}

Airado-Rodríguez, D., Durán-Merás, I., Galeano-Díaz, T., \& Wold, J. P. (2011). Frontface fluorescence spectroscopy: A new tool for control in the wine industry. Journal of Food Composition and Analysis, 24, 257-264.

Airado-Rodríguez, D., Galeano-Díaz, T., Durán-Merás, I., \& Wold, J. P. (2009). Usefulness of fluorescence excitation-emission matrices in combination with parafac, as fingerprints of red wines. Journal of Agricultural and Food Chemistry, $57,1711-1720$

Andersen, C. M., \& Bro, R. (2003). Practical aspects of PARAFAC modeling of fluorescence excitation-emission data. Journal of Chemometrics, 17, 200-215.

Candrawinata, V. I., Golding, J. B., Roach, P. D., \& Stathopoulos, C. E. (2013). From apple to juice-The fate of polyphenolic compounds. Food Reviews International, 29, 276-293.

Christensen, J., Nørgaard, L., Bro, R., \& Engelsen, S. B. (2006). Multivariate autofluorescence of intact food systems. Chemical Reviews, 106, 1979-1994.

Cohen, E., Birk, Y., Mannheim, C. H., \& Saguy, I. S. (1998). A rapid method to monitor quality of apple juice during thermal processing. LWT - Food Science and Technology, 31, 612-616.

Corradini, E., Foglia, P., Giansanti, P., Gubbiotti, R., Samperi, R., \& Laganà, A. (2011) Flavonoids: Chemical properties and analytical methodologies of identification and quantitation in foods and plants. Natural Product Research, 25, 469-495.

Craft, B. D., Kerrihard, A. L., Amarowicz, R., \& Pegg, R. B. (2012). Phenol-based antioxidants and the in vitro methods used for their assessment. Comprehensive Reviews in Food Science and Food Safety, 11, 148-173.

Feliciano, R. P., Antunes, C., Ramos, A., Serra, A. T., Figueira, M. E., Duarte, C. M. M., Carvalho, A., et al. (2010). Characterization of traditional and exotic apple varieties from portugal. Part 1 - Nutritional, phytochemical and sensory evaluation. Journal of Functional Foods, 2, 35-45.

Gardner, P. T., White, T. A. C., McPhail, D. B., \& Duthie, G. G. (2000). The relative contributions of vitamin $C$, carotenoids and phenolics to the antioxidant potential of fruit juices. Food Chemistry, 68, 471-474.

Giovanelli, G., Sinelli, N., Beghi, R., Guidetti, R., \& Casiraghi, E. (2014). NIR spectroscopy for the optimization of postharvest apple management Postharvest Biology and Technology, 87, 13-20.

Gliszczyńska-Świgło, A., \& Tyrakowska, B. (2003). Quality of commercial apple juices evaluated on the basis of the polyphenol content and the teac antioxidant activity. Journal of Food Science, 68, 1844-1849.

Gliszczyńska-Świgło, A., Ciska, E., Pawlak-Lemańska, K., Chmielewski, J., Borkowski, T., \& Tyrakowska, B. (2006). Changes in the content of health-promoting compounds and antioxidant activity of broccoli after domestic processing. Food Additives and Contaminants, 23, 1088-1098.

Ignat, I., Volf, I., \& Popa, V. I. (2011). A critical review of methods for characterisation of polyphenolic compounds in fruits and vegetables. Food Chemistry, 126, 1821-1835.

Kahle, K., Kraus, M., \& Richling, E. (2005). Polyphenol profiles of apple juices Molecular Nutrition and Food Research, 49, 797-806.

Kalinowska, M., Bielawska, A., Lewandowska-Siwkiewicz, H., Priebe, W., \& Lewandowski, W. (2014). Apples: Content of phenolic compounds vs. variety, part of apple and cultivation model, extraction of phenolic compounds, biological properties. Plant Physiology and Biochemistry, 84, 169-188.
Karaman, Ş., Tütem, E., Sözgen Başkan, K., \& Apak, R. (2010). Comparison of total antioxidant capacity and phenolic composition of some apple juices with combined HPLC-CUPRAC assay. Food Chemistry, 120, 1201-1209.

Karoui, R., Dufour, E., Bosset, J. O., \& De Baerdemaeker, J. (2007). The use of front face fluorescence spectroscopy to classify the botanical origin of honey samples produced in Switzerland. Food Chemistry, 101, 314-323.

Lenhardt, L., Bro, R., Zeković, I., Dramićanin, T., \& Dramićanin, M. D. (2015), Fluorescence spectroscopy coupled with PRAFAC and PLS-DA for characterization and classification of honey. Food Chemistry, 175, 284-291.

Lu, X., \& Rasco, B. A. (2011). Determination of antioxidant content and antioxidant activity in foods using infrared spectroscopy and chemometrics: A review. Critical Reviews in Food Science and Nutrition, 52, 853-875.

Mazina, J., Vaher, M., Kuhtinskaja, M., Poryvkina, L., \& Kaljurand, M. (2015). Fluorescence, electrophoretic and chromatographic fingerprints of herbal medicines and their comparative chemometric analysis. Talanta, 139, 233-246.

Mezzetti, A., Protti, S., Lapouge, C., \& Cornard, J.-P. (2011). Protic equilibria as the key factor of quercetin emission in solution. Relevance to biochemical and analytical studies. Physical Chemistry Chemical Physics, 13, 6858-6864.

Nicolaï, B. M., Beullens, K., Bobelyn, E., Peirs, A., Saeys, W., Theron, K. I., \& Lammertyn, J. (2007). Nondestructive measurement of fruit and vegetable quality by means of NIR spectroscopy: A review. Postharvest Biology and Technology, 46, 99-118.

Orzel, J., \& Daszykowski, M. (2014). A rapid validation of the antioxidant capacity of food commodities based on their fluorescence excitation emission spectra as applicable to coffee and peppermint extracts. Chemometrics and Intelligent Laboratory Systems, 137, 74-81.

Orzel, J., Stanimirova, I., Czarnik-Matusewicz, B., \& Daszykowski, M. (2015). Prediction of the hydrophilic antioxidant capacity of tomato pastes from the IR and fluorescence excitation-emission spectra of extracts and intact samples. Talanta, 138, 64-70.

Oszmiański, J., Wolniak, M., Wojdyło, A., \& Wawer, I. (2007). Comparative study of polyphenolic content and antiradical activity of cloudy and clear apple juices. Journal of the Science of Food and Agriculture, 87, 573-579.

Pissard, A., Fernández Pierna, J. A., Baeten, V., Sinnaeve, G., Lognay, G., Mouteau, A. Dupont, P., et al. (2013). Non-destructive measurement of vitamin C, total polyphenol and sugar content in apples using near-infrared spectroscopy. Journal of the Science of Food and Agriculture, 93, 238-244.

Poryvkina, L., Tsvetkova, N., \& Sobolev, I. (2014). Evaluation of apple juice quality using spectral fluorescence signatures. Food Chemistry, 152, 573-577.

Pyrzynska, K., \& Sentkowska, A. (2014). Recent developments in the HPLC separation of phenolic food compounds. Critical Reviews in Analytical Chemistry, 45, 41-51.

Re, R., Pellegrini, N., Proteggente, A., Pannala, A., Yang, M., \& Rice-Evans, C. (1999). Antioxidant activity applying an improved ABTS radical cation decolorization assay. Free Radical Biology and Medicine, 26, 1231-1237.

Robbins, R. J. (2003). Phenolic acids in foods: An overview of analytical methodology. Journal of Agricultural and Food Chemistry, 51, 2866-2887.

Schmutzler, M., \& Huck, C. W. (2014). Automatic sample rotation for simultaneous determination of geographical origin and quality characteristics of apples based on near infrared spectroscopy (NIRS). Vibrational Spectroscopy, 72, 97-104.

Seiden, P., Bro, R., Poll, L., \& Munck, L. (1996). Exploring fluorescence spectra of apple juice and their connection to quality parameters by chemometrics. Journal of Agricultural and Food Chemistry, 44, 3202-3205.

Sergiel, I., Pohl, P., Biesaga, M., \& Mironczyk, A. (2014). Suitability of threedimensional synchronous fluorescence spectroscopy for fingerprint analysis of honey samples with reference to their phenolic profiles. Food Chemistry, 145, 319-326.

Singleton, V. L., \& Rossi, J. A. (1965). Colorimetry of total phenolics with phosphomolybdic-phosphotungstic acid reagents. American Journal of Enology and Viticulture, 16, 144-158.

Tsimogiannis, D., Samiotaki, M., Panayotou, G., \& Oreopoulou, V. (2007). Characterization of flavonoid subgroups and hydroxy substitution by HPLCMS/MS. Molecules, 12, 593-606.

Wold, S., Sjöström, M., \& Eriksson, L. (2001). PLS-regression: A basic tool of chemometrics. Chemometrics and Intelligent Laboratory Systems, 58, 109-130.

Wu, J., Gao, H., Zhao, L., Liao, X., Chen, F., Wang, Z., \& Hu, X. (2007). Chemical compositional characterization of some apple cultivars. Food Chemistry, 103, 88-93.

Wulf, J. S., Rühmann, S., Rego, I., Puhl, I., Treutter, D., \& Zude, M. (2008). Nondestructive application of laser-induced fluorescence spectroscopy for quantitative analyses of phenolic compounds in strawberry fruits (fragaria $x$ ananassa). Journal of Agricultural and Food Chemistry, 56, 2875-2882.

Zhu, D., Ji, B., Eum, H. L., \& Zude, M. (2009). Evaluation of the non-enzymatic browning in thermally processed apple juice by front-face fluorescence spectroscopy. Food Chemistry, 113, 272-279.

Žiak, Ľ., Sádecká, J., Májek, P., \& Hroboňová, K. (2014). Simultaneous determination of phenolic acids and scopoletin in brandies using synchronous fluorescence spectrometry coupled with partial least squares. Food Analytical Methods, 7 , 563-570. 